\section{Arresting infant retinopathy}

\section{By Brian Moy, Staff Writer}

Researchers at the Cleveland Clinic have found that activating hypoxia-inducible factor can prevent the progression of retinopathy in premature infants, which results in blindness if left unchecked. The findings, reported in the Proceedings of the National Academy of Sciences, open a new window for treating retinopathy of prematurity, although the challenge will be to pinpoint the proper time frame for administering HIF activators.

Retinopathy of prematurity (ROP), which affects more than $75 \%$ of severely premature infants, has two phases, which are based on the oxygen-regulated expression of VEGF. ${ }^{1}$ In the initial hyperoxic phase of the disease, decreases in VEGF lead to oxygen-induced vascular obliteration of the developing retina.

In the second, hypoxic, phase of the disease, the consequent lack of blood supply to the retina leads to vast areas of ischemia that prompt the overexpression of VEGF and erythropoietin (EPO). This results in pathologic neovascularization that causes bleeding and retinal detachment.

The Cleveland Clinic researchers sought to simulate hypoxia during the hyperoxic first phase by inhibiting hypoxia-inducible factor prolyl hydroxylase (EGLN2; HIF-PH; PHD). They hypothesized that the approach could prevent development of hypoxia-induced neovascularization and progression to the proliferative stage of the disease by promoting more robust upregulation of the proangiogenic molecules VEGF and EPO. ${ }^{2}$

PHD induces hydroxylation of hypoxia-inducible factor $1 \alpha$ (HIF1A; HIF-1 $\alpha$ ), which leads to the degradation and decreased stability of the HIF- $1 \alpha$ transcription factor. Depending on the concentration of oxygen within the cell, HIF- $1 \alpha$ either is rapidly ubiquitinated and degraded or becomes stabilized to transcribe various hypoxia-responsive genes, such as VEGF and $E P O$ (see Figure 1, "HIF regulation of the body's response to oxygen concentration”).

In the PNAS paper, the researchers treated a mouse model of ROP with the PHD inhibitor dimethyloxalylglycine (DMOG) during the hyperoxic phase. The treatment prevented oxygen-induced vessel loss and led to significant decreases in vascular pathology compared with what was seen in saline-treated controls.

Compared with control mice, systemic injection of DMOG at postnatal day 6 and 8 led to significant decreases in capillary dropout $(p=0.004)$, in blood vessel dilation around the optic nerve $(p=0.043)$ and in abnormal vessel growth $\left(p=5.7 \times 10^{-7}\right)$.
On the other hand, administration of DMOG during the hypoxic phase of the disease at postnatal day 11 and 13 did not induce protective effects.

"The idea behind our study was that if we can prevent blood vessels from dying, we can prevent ischemia associated with phase II of ROP from occurring," Jonathan Sears, principal investigator of the study and an associate professor of ophthalmology at the Cole Eye Institute at Cleveland Clinic, told SciBX. "Our findings demonstrate that the use of small molecule activators of HIF can regulate the normal, sequential development of blood vessels by overriding hyperoxia-induced vascular obliteration."

As anticipated, activating HIF in the mice under hyperoxic conditions caused upregulation of Epo and Vegf that prevented vascular loss, ischemia and subsequent proliferative retinopathy.

Sears also noted that Hif- $1 \alpha$ stability increased in the liver of mice treated with DMOG. "This suggests that the fetal liver may be the key to regulating angiogenesis in capillary beds distant from the liver," he said. Capillary beds are networks of small blood vessels or capillaries that supply nutrients to an organ, such as the eyes.

The researchers wrote that the "DMOG-mediated increase in HIF- $1 \alpha$ stability is liver specific and regulates downstream genes such as VEGF and EPO in a paracrine and endocrine manner to protect the retinal vasculature."

The upshot, said Sears, is that "perhaps a soluble pill or subcutaneous injection of a HIF- $1 \alpha$ activator into the liver could be used to promote protection of capillary beds for treating ROP," rather than using a direct injection into the eye.

\section{Timing matters}

Although the findings in the PNAS article provide a new strategy for developing therapies for ROP, researchers cautioned that administration during the proliferative stage of the disease might exacerbate pathology.

According to Martin Friedlander, a professor of cell biology at The Scripps Research Institute and chief of the retina service at Scripps Clinic, "the timing of the dosing is critical. If the HIF- $1 \alpha$ activator is administered at the wrong time, it could ultimately enhance neovascularization and lead to disease progression."

He added that because HIF- $1 \alpha$ initiates a complex cascade of events in response to oxygen concentration, "it will be important to determine exactly what role the transcription factor plays in the angiogenic process before moving forward."

According to Sears, the key next step will be to determine the proper timing of administering HIF- $1 \alpha$ activators to safely affect the physiologic expression of the gene products that regulate the body's response to hypoxia.

Ryo Kubota, CEO of Acucela Inc., agreed. "Because the therapeutic window of dosing during the hyperoxic phase of ROP is relatively narrow, additional preclinical testing with HIF- $1 \alpha$ activators is needed to determine the optimal time to administer such compounds," he said. 


\section{TARGETS \& MECHANISMS}

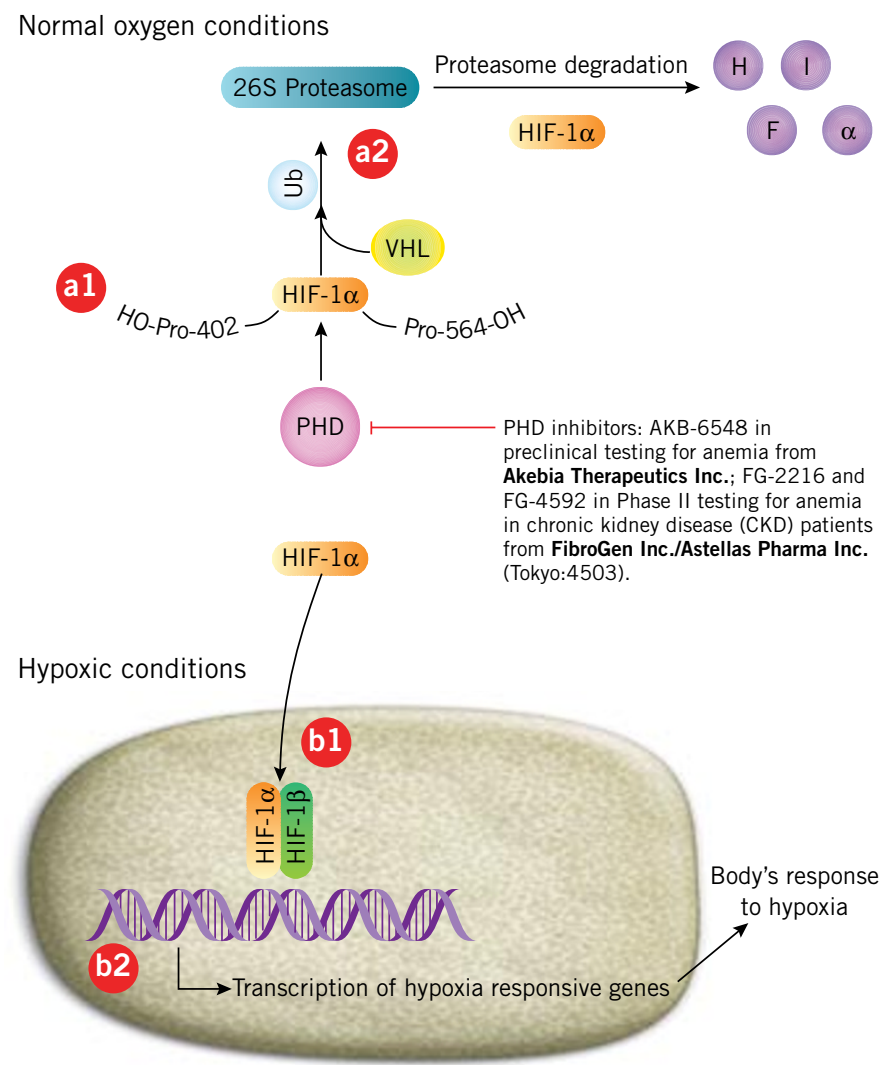

Kubota also noted that because HIF- $1 \alpha$ ultimately regulates angiogenesis, "future research should show that treatment will not lead to other angiogenic side effects or unwanted vascular remodeling. This is especially important because the drugs will ultimately be used to treat disease in premature infants."

Last September, Acucela partnered with Otsuka Pharmaceutical Co. Ltd. to develop the biotech's ACU-4429 for dry age-related macular degeneration (AMD) and other indications such as diabetic retinopathy. The small molecule nonretinoid visual cycle modulator is in Phase I testing.

Ongoing studies by Sears and colleagues are investigating the correct dose and timing of administration of HIF- $1 \alpha$ activators. The researchers are identifying additional small molecule activators of HIF and inhibitors of PHD.

Sears told SciBX the findings from the PNAS paper are neither patented nor licensed.

PHD inhibitors in development include: AKB-6548, an oral PHD inhibitor from Akebia Therapeutics Inc. that is in preclinical testing
Figure 1. HIF regulation of the body's response to oxygen concentration. Inhibiting hypoxia-inducible factor prolyl hydroxylase (EGLN2; HIF-PH; PHD) could prevent the development of hypoxia-induced neovascularization and progression to the proliferative stage of retinopathy of prematurity (ROP). Depending on the concentration of oxygen within the cell, hypoxia-inducible factor $1 \alpha$ (HIF1A; HIF-1 $\alpha$ ) either is rapidly ubiquitinated (Ub) and degraded or becomes stabilized to transcribe various hypoxiaresponsive genes.

Under normal oxygen conditions, PHD induces hydroxylation of two proline residues (Pro-402 and Pro-564) on HIF-1 $\alpha$ [a1]. Hydroxylated HIF-1 $\alpha$ is then recognized by the von HippelLindau (VHL) tumor suppressor protein to promote ubiquitination and degradation of HIF- $1 \alpha$ in the $26 \mathrm{~S}$ proteasome [a2].

In contrast, under hypoxic conditions, PHD is suppressed and HIF- $1 \alpha$ dimerizes with HIF- $1 \beta$ to form an active HIF complex within the nucleus [b1]. The heterodimer transcribes specific DNA sequences that encode hypoxia-responsive genes. These genes encode proteins that play a key role in the body's response to hypoxia by preventing vascular loss and ischemia, thus preventing progression to the proliferative stage of ROP [b2].

There are already compounds in development that target PHD for anemia.

for anemia, and FG-2216 and FG-4592, small molecule PHD inhibitors from FibroGen Inc. and Astellas Pharma Inc. that are in Phase II testing for anemia in chronic kidney disease (CKD) patients.

Moy, B. SciBX 2(2); doi:10.1038/scibx.2009.40

Published online Jan. 15, 2009

\section{REFERENCES}

1. Alon, T. et al. Nat. Med. 1, 1024-1028 (1995)

2. Sears, J. et al. Proc. Natl. Acad. Sci. USA; published online Dec. 1, 2008; doi:10.1073/pnas.0805817105 Contact: Jonathan E. Sears, Cleveland Clinic, Cleveland, Ohio e-mail: searsj@ccf.org

\section{COMPANIES AND INSTITUTIONS MENTIONED}

Acucela Inc., Bothell, Wash.

Akebia Therapeutics Inc., Cincinnati, Ohio

Astellas Pharma Inc. (Tokyo:4503), Tokyo, Japan

Cleveland Clinic, Cleveland, Ohio

FibroGen Inc., San Francisco, Calif.

Otsuka Pharmaceutical Co. Ltd. (Tokyo:4768), Tokyo, Japan

The Scripps Research Institute, La Jolla, Calif. 